\title{
OPEN Inactivation of genes in oxidative respiration and iron acquisition pathways in pediatric clinical isolates of Small colony variant Enterobacteriaceae
}

\begin{abstract}
Alexander L. Greninger ${ }^{1,2,4}$, Amin Addetia ${ }^{1,2,4}$, Yue Tao ${ }^{3}$, Amanda Adler ${ }^{2}$ \& Xuan Qin $^{1,2} \bowtie$
Isolation of bacterial small colony variants (SCVs) from clinical specimens is not uncommon and can fundamentally change the outcome of the associated infections. Bacterial SCVs often emerge with their normal colony phenotype (NCV) co-isolates in the same sample. The basis of SCV emergence in vivo is not well understood in Gram-negative bacteria. In this study, we interrogated the causal genetic lesions of SCV growth in three pairs of NCV and SCV co-isolates of Escherichia coli, Citrobacter freundii, and Enterobacter hormaechei. We confirmed SCV emergence was attributed to limited genomic mutations: 4 single nucleotide variants in the $E$. coliSCV, 5 in $C$. freundii, and 8 in $E$. hormaechei. In addition, a $10.2 \mathrm{~kb}$ chromosomal segment containing 11 genes was deleted in the $E$. hormaechei SCV isolate. Each SCV had at least one coding change in a gene associated with bacterial oxidative respiration and another involved in iron capture. Chemical and genetic rescue confirmed defects in heme biosynthesis for $E$. coli and $C$. freundii and lipoic acid biosynthesis in $E$. hormaachei were responsible for the SCV phenotype. Prototrophic growth in all 3 SCV Enterobacteriaceae species was unaffected under anaerobic culture conditions in vitro, illustrating how SCVs may persist in vivo.
\end{abstract}

To survive in the hostile host environment, bacteria may take two separate paths. The first and most commonly discussed is an arms race of iron competition and acquisition of antimicrobial resistance genes and pathogenicity factors $^{1-3}$. The alternative path is to adapt to persist through reductions in metabolic needs and an attenuated growth rate. The isolates that display this alternative phenotype are known as small colony variants (SCVs). Bacterial SCVs were first described in Salmonella typhi over a hundred years ago, prior to the antibiotic era ${ }^{4}$. Isolation of SCVs is especially common in recurrent or persistent infections involving the respiratory tract, urinary tract, mid-ear, foreign body-related implants, and bone and joint ${ }^{5-7}$. Emergence of bacterial SCVs from normal colony phenotype (NCV) parental isolates has been described in various clinical settings ${ }^{4}$. Previously characterized bacterial SCV species include Staphylococcus aureus, Escherichia coli, Neisseria gonorrhoeae, Stenotrophomonas maltophilia, Enterococcus, and Salmonella ${ }^{8-12}$. In addition to their decreased growth rate, bacterial SCV isolates are characterized by auxotrophism for components directly involved in the electron transfer chain, such as heme and menaquinone. SCVs may also display a nutritional dependency on thymidine or methionine ${ }^{8,13}$. From a treatment perspective, SCVs display a reduced response to antibiotics despite not carrying the associated antimicrobial resistance genes ${ }^{4,6,14,15}$. These degenerative changes allow SCVs to persist in vivo in the unique environment and selection pressures of the infected host.

SCVs have been best characterized among S. aureus isolates. Common gene lesions in S. aureus SCVs have been seen in the heme, menaquinone, and thymidine biosynthetic pathways ${ }^{6,7}$. In clinical strains, disruptions in menaquinone biosynthesis have been associated with mutations in men $B$, menC, menE and $m e n F^{16}$. Laboratory-derived SCV S. aureus, Salmonella typhimurium, or E. coli with hemA, hemB, hemD, hemL, lipA, or ctaA mutations have been used for functional characterization of their changes in growth, metabolism, antimicrobial susceptibility, host invasion, and persistence ${ }^{17-21}$. In addition, mutations in transcriptional regulators in

${ }^{1}$ Department of Laboratory Medicine and Pathology, University of Washington, Seattle, WA, USA. ${ }^{2}$ Seattle Children's Hospital, Seattle, WA, USA. ${ }^{3}$ Shanghai Children's Medical Center, Translational Research Institute, Shanghai Jiao Tong University School of Medicine, Shanghai 200127, China. ${ }^{4}$ These authors contributed equally: Alexander L. Greninger and Amin Addetia. ${ }^{\varpi}$ email: qinxu@ohsu.edu 
Staphylococcus spp. governing bacterial virulence factor expression, such as agr, sarA, sigB, and relA, have also been detected, suggesting attenuated cytotoxicity may enable bacterial persistence ${ }^{7,15,22,23}$. However, the basis of SCV formation and persistence in clinical isolates of Gram-negative bacteria has been less well characterized ${ }^{10,24}$.

Laboratory recognition, isolation, characterization and appropriate report of bacterial SCVs have suffered from a lack of established standards or guidelines. We previously reported a method practical in clinical laboratories for recognition and phenotypic characterization of SCV S. maltophilia from airway secretions of CF patients $^{8}$. Our lab has since implemented a systematic, culture-based approach that checks not only for colony variation in size, texture, color, or hemolysis, but also inability to grow on the standard Mueller-Hinton (MH) medium for susceptibility testing. Using this systematic approach, we identified 3 pairs of clinical NCV and SCV Enterobacteriaceae co-isolates from blood and urine cultures. We then used whole genome sequencing to screen for the molecular mechanisms distinguishing the SCVs from their NCV co-isolates. Confirmatory chemical and genetic rescues were performed on the SCVs to determine which mutations were causal for the altered phenotype.

\section{Methods}

Isolation and characterization of SCV and NCV from clinical samples. This study was approved by Seattle Children's Hospital Institutional Review Board with a consent waiver. Three clinical NCV and SCV co-isolate pairs of Escherichia coli, Citrobacter freundii, and Enterobacter hormaechei were obtained from urine or blood cultures. Colony variants were separated by sub-cultures and bacterial species identification was performed using MALDI-TOF (Bruker biotyper, Bruker Daltonics, Inc.). Of note, the SCVs isolates described here did not grow on Mueller-Hinton agar and, thus, were reported in the patient's clinical record without antimicrobial susceptibility.

Case histories. Case 1-A previously healthy 6-week-old male with no history of hospitalization or receipt of antibiotics, presented to the emergency department with fever. His white blood cell count was elevated at $21,600 / \mathrm{ml}$ and a $2+$ urine leukocyte esterase at the time of emergency visit. The patient was admitted for a rule-out sepsis workup and the patient was started on empiric ceftriaxone. Urine cultures grew $10^{3}-10^{4} \mathrm{cfu} / \mathrm{mL}$ Escherichia coli and $10^{3}-10^{4} \mathrm{cfu} / \mathrm{mL}$ SCV Escherichia coli. It was felt the urine culture did not support the diagnosis of UTI, and no additional antibiotics were indicated. The patient recovered fully.

Case 2-A 2-month-old female with right duplicated collection system presented to the emergency department with fever and foul-smelling urine. The patient had experienced two urinary tract infections (UTIs) in the previous month (Escherichia coli and Citrobacter spp.) that were treated with amoxicillin and cephalexin, respectively. Given that the patient was currently receiving antibiotics for the previous Gram-negative UTI, the decision was made to admit the patient for likely IV antibiotic treatment, and the patient was started on empiric piperacillin-tazobactam. Her urine leukocyte esterase was $3+$ with elevated red and white blood cells in urine at the time of culture. Urine cultures grew $>10^{5} \mathrm{cfu} / \mathrm{mL}$ Citrobacter spp. and $5 \times 10^{4}-10^{5} \mathrm{cfu} / \mathrm{mL}$ SCV Citrobacter spp. and therapy was switched to ciprofloxacin. The patient completed 14 days of therapy and recovered fully.

Case 3-A 6 year-old male with end stage renal disease managed with renal dialysis presented to dialysis clinic with fever, hypotension, and tachycardia. The patient had a history of multiple bloodstream infections treated with ceftazidime, rifampin, gentamicin, or intravenous trimethoprim-sulfamethoxazole. The patient was admitted to the hospital and started on empiric vancomycin and gentamicin. Blood cultures grew out Enterobacter cloacae and SCV Enterobacter cloacae by MALDI-TOF and therapy was switched to cefepime. Additionally, the patient received ceftazidime line-lock therapy. The patient completed treatment and recovered fully. Though the isolates were resulted out as E. cloacae, they were later identified as E. hormaechei based on whole genome sequencing.

Whole genome sequencing of isolates. Whole genome sequencing and assembly of the resulting sequencing reads for the NCV and SCV co-isolates were performed as previously described ${ }^{25,26}$. Briefly, DNA was extracted from using the MoBio UltraClean Microbial DNA Isolation kit. DNA was diluted to $1 \mathrm{ng} / \mathrm{uL}$ and tagmented using quarter-volume reactions of Nextera XT with 15 cycles of PCR amplification. Libraries were sequenced on a $2 \times 300$ bp run on an Illumina MiSeq to achieve $>1$ million paired-end reads per sample. Reads were quality and adapter-trimmed using cutadapt (Q30) and de novo assembled using SPAdes using default parameters. For each pair of isolates, the NCV assembly was annotated using prokka and the reads from the paired isolate were mapped to the annotated assembly in Geneious v9.1. Variants were called using a minimum coverage of $7 \mathrm{X}$ and a minimum allele frequency of $75 \%{ }^{27}$. All variants were manually curated and variants within $10 \mathrm{bp}$ of the edge of a contig were removed.

Recombinant bacterial strains, cloning, and plasmid preparation. The ASKA library is a comprehensive GFP-tagged E. coli K-12 ORF plasmid library available from the National BioResource Project. The ASKA clone library is based on the E. coli K-12 strain AG1 and individual genes were cloned into the pCA24N vector $^{28}$. The E. coli strain K-12 carrying pCA24N::hemL, hemN, hemF, fes, fepC, araC, cysD, udk, pta, mprA, cusA, cusB, cusF, cspE, crcB, tatE, lipA, entD, tolC, rnr, hyfB and pCA24N plasmid itself were obtained from the National BioResource Project. These E. coli strains were grown at $35^{\circ} \mathrm{C}$ for $18 \mathrm{~h}$ in TSY broth (Remel) with chloramphenicol $(50 \mu \mathrm{g} / \mathrm{ml})$. Plasmids were isolated using the ZymoPure Plasmid MiniPrep kit (Zymo Research) and then transformed into the clinical strains of E.coli, C.freundii, and E. hormaechei as described below.

One of the affected genes, $p q q B$, in the SCV E. hormaechei isolates was unavailable from the ASKA strain library as an ortholog of this gene is not present in E. coli K-12. This gene was amplified from the NCV E. hormaechei isolate with CloneAmp HiFi polymerase (Takara) and the following primers: 5'-TCC GGC CCT GAG GCC TAT GGC CTT TAT TAA AGT CCT CGG TTC C-3' and 5'-TCC TTT ACT TGC GGC CGG GGT CCT GAA GCG TGA TGT TCA T-3'. These PCR products were cloned into pCA24N with a C-terminal GFP tag using the 
In-Fusion HD enzyme kit (Takara). Clones were selected on TSA plates with $50 \mu \mathrm{g} / \mathrm{ml}$ chloramphenicol. Sanger sequencing was conducted on the resulting plasmid to confirm cloning.

Preparation of competent cells and electroporation. To prepare electro-competent cells of E.coli, C.freundii, and E. hormaechei, $5 \mathrm{~mL}$ of TSY broth inoculated with a single colony was grown overnight with vigorous aeration $(150 \mathrm{rpm} / \mathrm{min})$ at $35^{\circ} \mathrm{C}$. The following day, $30 \mu \mathrm{l}$ of overnight culture was diluted into $15 \mathrm{ml}$ of SOC media and grown at $37^{\circ} \mathrm{C}$ with constant shaking $(180 \mathrm{rpm} / \mathrm{min})$ until $0.5-0.8$ OD600. Cells were harvested by centrifugation at $2000 \mathrm{~g}$ for $10 \mathrm{~min}$ at $4{ }^{\circ} \mathrm{C}$ and washed twice with $10 \mathrm{ml}$ sterile ice-cold $10 \%$ glycerol. The supernatant was removed and the cell suspension was concentrated 50 -fold in $3 \%$ glycerol.

For bacterial transformation, $100 \mathrm{uL}$ of electrocompetent cells were mixed with $100 \mathrm{ng}$ DNA in $0.1 \mathrm{~cm}$ cuvettes. Electroporation was carried out using a Gene Pulser with the following parameters: $2.5 \mathrm{kV}, 25 \mu \mathrm{F}$ and $200 \Omega$ for the NCV and SCV E. coli, NCV and SCV E. hormaechei and SCV C. freundii. The following parameters were used to transform the NCV C. freundii: $2.5 \mathrm{kV}, 25 \mu \mathrm{F}$ and $600 \Omega$. Immediately after pulsing, $0.9 \mathrm{ml}$ of SOC media was added to each cuvette, the cell suspension was transferred to a test tube and then was incubated for $30 \mathrm{~min}$ at $37^{\circ} \mathrm{C}$ with constant shaking. Transformed clones were selected on TSA plates with $50 \mu \mathrm{g} /$ $\mathrm{ml}$ chloramphenicol.

Chemical and genetic rescue and cross-feeding. Unlike their NCV co-isolates, SCVs cannot grow on M9 minimal media. We took advantage of this to study the additional nutritional requirements of SCVs and determine which mutations were casual for the auxotrophic phenotypes. To study the nutritional requirements of each SCV, a 0.5 McFarland solution of the isolate was plated on M9 media. A disk impregnated with heme (Remel), $\delta$-aminolevulinic acid (Oxoid), L-glutamate (Sigma-Aldrich, $30 \mathrm{mM}$ ), lipoic acid (Sigma-Aldrich, $5 \mu \mathrm{g} / \mathrm{mL}$ ) or pyrroloquinoline quinone (Sigma-Aldrich, $3 \mu \mathrm{M}$ ) was placed onto the media. For each chemical rescue, the corresponding NCV co-isolate and a blank disk were included as controls. The M9 plates were examined after incubation for $20-24 \mathrm{~h}$ at $35^{\circ} \mathrm{C}$. Of note, NCV and SCV C. freundii carrying pCA24N were used in place of the untransformed NCV and SCV C. freundii due to the instability of the SCV isolate.

To examine the causal mutations responsible for the SCV phenotype, $0.5 \mathrm{McF}$ arland solutions of each of the complemented strains were plated on to M9 media (Teknova). The M9 plates were examined after incubation for $20-24 \mathrm{~h}$ at $35^{\circ} \mathrm{C}$. We examined whether the NCV could restore the growth of its SCV co-isolates by growing the strains adjacent to one another on M9 media. A $0.5 \mathrm{McF}$ arland standard of each isolate was streaked on an M9 agar plate. This plate was examined after incubation for $20-24 \mathrm{~h}$ at $35^{\circ} \mathrm{C}$. Anaerobic cultures were also performed at $35^{\circ} \mathrm{C}$ using the AnaeroPack system (Mitsubishi Gas Chemical) and growth was observed at $48 \mathrm{~h}$.

Aerobic and anaerobic growth curves. Growth kinetics of NCV and SCV co-isolates, along with genetically rescued SCV isolates, were assessed in aerobic and anaerobic conditions. For aerobic growth curves, overnight cultures were diluted in a microplate containing $200 \mu \mathrm{L}$ of Luria-Bertani (LB) broth (Gibco) such that the starting $\mathrm{OD}_{600}$ of the culture was approximately 0.01 . The microplate was then incubated at $37{ }^{\circ} \mathrm{C}$ and $\mathrm{OD}_{600}$ readings were taken every 15 min using SpectraMax 190 operated with the SpectraMax Pro software version 4.3. A 10-s shaking was completed prior to each reading. For anaerobic growth curves, overnight cultures from each strain were diluted into four tubes containing $5 \mathrm{~mL}$ of pre-reduced $\mathrm{LB}$ broth to an $\mathrm{OD}_{600}$ of approximately 0.01 . The cultures were then incubated at $35^{\circ} \mathrm{C}$ in a sealed container containing an AnaeroPack anaerobic gas generator. Every $1.5 \mathrm{~h}, \mathrm{OD}_{600}$ measurements were taken using one of the four initially noculated cultures. Aerobic growth curves were completed in biological triplicate and anaerobic growth curves were completed in biological duplicate for each strain.

\section{Results}

Clinical cases and isolates. Case histories are depicted in Fig. 1 and described in the Methods. Briefly, the paired E. coli isolates were from a urine culture on a 6-week old otherwise healthy term male infant with fever. His white blood cell count was elevated at $21,600 / \mathrm{ml}$ and a $2+$ urine leukocyte esterase at the time of emergency visit. The $C$. freundii isolates were from a urine culture on a 2-month old female infant with complex urological anomalies for duplicated collecting system and grade 3 vesicoureteral reflux. Her urine leukocyte esterase was $3+$ with elevated red and white blood cells in urine at the time of culture. The E. hormaechei isolates were from multiple blood cultures, both arterial line and peripheral venous draw, spanning 3 days on a 6-year old male child with end stage renal disease receiving hemodialysis and multiple prior bloodstream infections in the prior year. Of note, these isolates were originally resulted out as E. cloacae based on MALDI-TOF species identification.

Antimicrobial resistance pattern is explained by ampC. Co-isolation of both NCV and SCV of the Enterobacteriaceae strains were common to all 3 cultures during routine culture workups. The antibiotic susceptibility patterns for the three NCV isolates are shown in Table 1 and followed expected patterns of resistance given the case histories. All three corresponding SCV isolates failed to grow on MH medium for susceptibility testing.

Genomic sequencing of the NCV isolates confirmed the presence of chromosomal ampC in all isolates, explaining the antibiotic susceptibility patterns recovered. Analysis of contigs with higher copy number revealed one small $4 \mathrm{~kb}$ plasmid in Escherichia coli, three small 2.5-4 kb plasmids in Enterobacter hormachei, as well as $>80 \mathrm{~kb}$ of phage sequence in the Citrobacter freundii assembly but no plasmids. No specific antimicrobial resistance genes were contained on these plasmids. 
a Case 1

SCV \& NCV

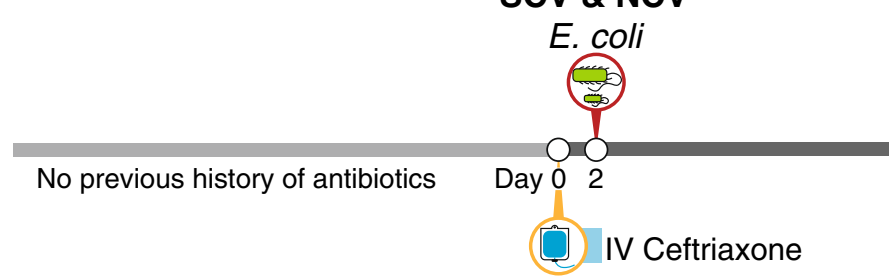

b Case 2

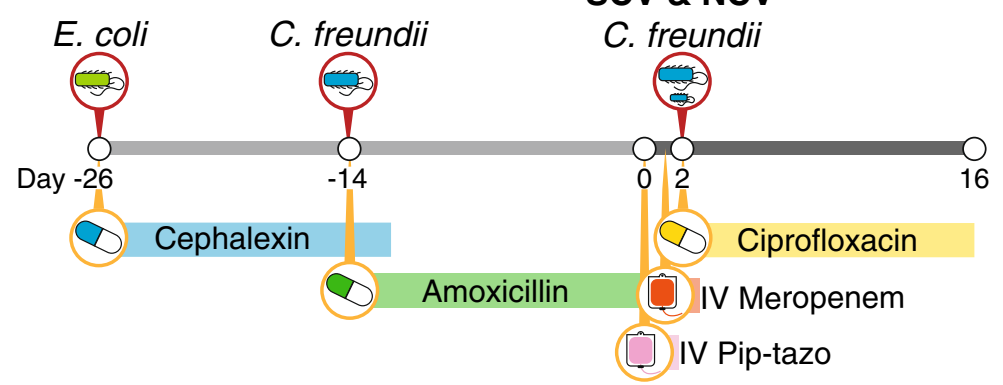

c Case 3

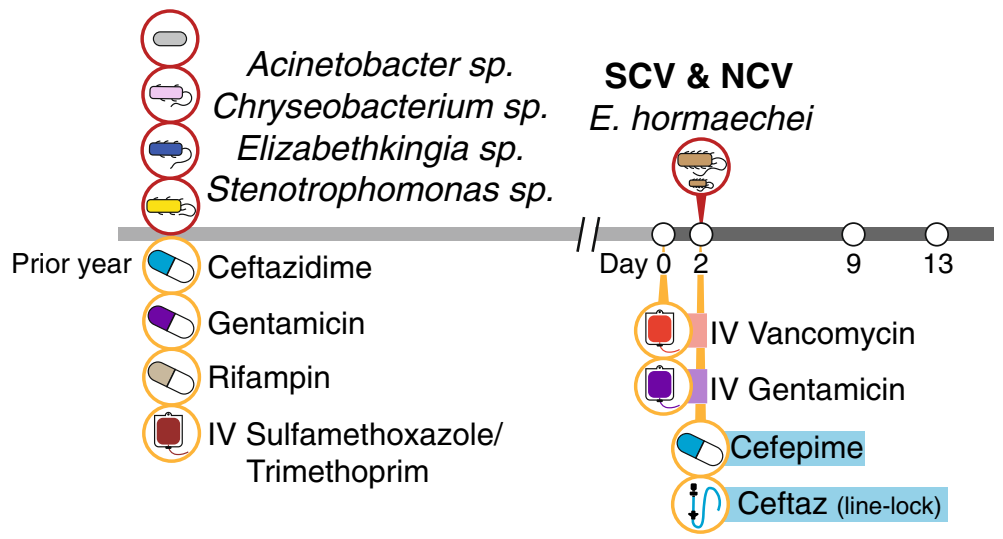

Figure 1. Case histories. Relevant past clinical microbiological and antibiotic selective pressures are indicated in the line histories for the isolation of NCV and SCV in Escherichia coli (a), Citrobacter freundii (b), and Enterobacter hormaechei (c).

Whole genome sequencing of paired isolates reveals parsimonious variants accounting for small colony phenotype. The Escherichia coli NCV assembly yielded 81 contigs $>200$ bp with an N50 of $360,223 \mathrm{bp}$. Mapping of the Escherichia coli SCV reads to the NCV assembly yielded only 4 variants, including 2 variants in heme-related genes (Table 2). The 272-amino acid enterobactin siderophore transport system ATPbinding protein $(f e p C)$ gene had an internal stop codon at amino acid 169. The hemL gene had a W59R coding change and the hemF gene had an in-frame 6-bp deletion resulting in the loss of an arginine and glutamic acid at amino acids 132 and 133. In addition, the transcriptional repressor $m p r A$ gene had a P84S coding change. No intergenic variants were recovered in the Escherichia coli SCV strain relative to the NCV strain.

Sequencing the Citrobacter freundii NCV strain yielded an assembly of 220 contigs $>200$ bp with an N50 of 61,653 bp. Mapping of the Citrobacter freundii SCV reads to the NCV assembly yielded a total of 6 variants (Table 2). Two of these variants were related to heme-producing genes. Most notable among these were a 1 bp deletion in the glutamate-1-semialdehyde 2,1-aminomutatase (hemL) gene that resulted in frame shift and stop codon at amino acid 75, as well as a premature stop codon at amino acid 918 of the enterochelin esterase (fes) gene. An additional heme-related variant included an intergenic mutation of unclear significance, $181 \mathrm{bp}$ upstream of the aerobic coproprohphyrinogen-III oxidase (hemF) gene. The three remaining variants resulted in coding changes in genes unrelated to heme production, including a Q303L mutation in the phosphate acetyltransferase ( $p t a)$ gene, a P778T mutation in the major subunit precursor of the nitrate-inducible formate dehydrogenase $(f d n G)$ gene, and a P7L mutation in an arabinose operon regulatory protein (araC) gene. 


\begin{tabular}{|l|l|l|l|l|l|l|}
\hline \multirow{2}{*}{ Disk content } & \multicolumn{3}{|l|}{ Zone diameter } & Escherichia coli & Citrobacter freundii & Enterobacter hormaechei \\
\cline { 2 - 8 } & $\mathbf{S}^{*}$ & $\mathbf{I}^{*}$ & $\mathbf{R}^{*}$ & Result $(\mathbf{m m})$ & Result $(\mathbf{m m})$ & Result $(\mathbf{m m})$ \\
\hline Ampicillin $(10 \mu \mathrm{g})$ & $\geq 17$ & $14-16$ & $\leq 13$ & $\mathrm{R}(15)$ & $\mathrm{R}(6)$ & $\mathrm{R}(6)$ \\
\hline Augmentin $(20 / 10 \mu \mathrm{g})$ & $\geq 18$ & $14-17$ & $\leq 13$ & $\mathrm{I}(17)$ & $\mathrm{R}(7)$ & $\mathrm{R}(10)$ \\
\hline Cefazolin & $\geq 23$ & $20-22$ & $\leq 19$ & $\mathrm{I}(21)$ & $\mathrm{R}(6)$ & $\mathrm{R}(6)$ \\
\hline Cefazolin (urine breakpoint) & $\geq 15$ & & $\leq 14$ & $\mathrm{~S}(21)$ & $\mathrm{R}(6)$ & $\mathrm{R}(6)$ \\
\hline Ceftazidime & $\geq 21$ & $18-20$ & $\leq 17$ & $\mathrm{~S}(31)$ & $\mathrm{R}(14)$ & $\mathrm{S}(27)$ \\
\hline Ceftriaxone & $\geq 23$ & $20-22$ & $\leq 19$ & $\mathrm{~S}(32)$ & $\mathrm{R}(16)$ & $\mathrm{S}(27)$ \\
\hline Cefuroxime (IV) & $\geq 18$ & $14-17$ & $\leq 13$ & $\mathrm{~S}(20)$ & $\mathrm{R}(8)$ & $\mathrm{R}(6)$ \\
\hline Cefepime & $\geq 25$ & $19-24$ & $\leq 18$ & $\mathrm{~S}(33)$ & $\mathrm{S}(32)$ & $\mathrm{S}(33)$ \\
\hline Piperacillin-tazobactam & $\geq 21$ & $18-20$ & $\leq 17$ & $\mathrm{~S}(28)$ & $\mathrm{S}(21)$ & $\mathrm{S}(27)$ \\
\hline Meropenem & $\geq 23$ & $20-22$ & $\leq 19$ & $\mathrm{~S}(30)$ & $\mathrm{S}(29)$ & $\mathrm{S}(28)$ \\
\hline Ciprofloxacin & $\geq 31$ & $21-30$ & $\leq 20$ & $\mathrm{~S}(33)$ & $\mathrm{I}(23)$ & $\mathrm{S}(34)$ \\
\hline Gentamicin & $\geq 15$ & $13-14$ & $\leq 12$ & $\mathrm{~S}(23)$ & $\mathrm{S}(23)$ & $\mathrm{S}(20)$ \\
\hline Nitrofurantoin (urine breakpoint) & $\geq 17$ & $15-16$ & $\leq 14$ & $\mathrm{~S}(26)$ & $\mathrm{S}(21)$ & $\mathrm{N} . \mathrm{D}$ \\
\hline Trimethoprim-sulfamethoxazole & $\geq 16$ & $11-15$ & $\leq 10$ & $\mathrm{~S}(29)$ & $\mathrm{S}(27)$ & $\mathrm{S}(25)$ \\
\hline
\end{tabular}

Table 1. Antibiotic susceptibility by disk diffusion of normal colony variant for each isolate based on CLSI M100-S29 breakpoints. *"S" sensitive, "I" intermediate, and "R" resistant.

The Enterobacter hormaechei NCV assembly yielded 60 contigs longer than $200 \mathrm{bp}$. Mapping of the Enterobacter hormaechei SCV reads to the NCV assembly yielded 7 single nucleotide coding variants, one of which was in a gene related to heme production and one involved in enterobactin synthesis. The oxygen-independent coproporphyrinogen-III oxidase (hem N) gene had a L366Q mutation, while the 4'-phosphopantetheinyl transferase (entD) involved in enterobactin synthesis complex had a D21A mutation. Multiple unrelated coding changes were found between the SCV and the NCV Enterobacter hormaechei strains, including a G291S mutation in the sulfate adenylyltransferase subunit $2(c y s D)$ gene, a C224W mutation in the coenzyme PQQ synthesis protein $\mathrm{B}(p q q B)$, a I362T mutation in the sensor histidine kinase $(r c s C)$, a T403S mutation in the autoinducer 2 kinase $(l s r K)$, and a $\mathrm{D} 74 \mathrm{H}$ mutation in uridine kinase $(u d k)$. An intergenic G- $>$ A mutation 45 bp downstream of a hypothetical protein was also identified. A $10.2 \mathrm{~kb}$ chromosomal deletion disrupting 11 genes was also found in the SCV assembly as compared to the NCV assembly. These genes included lipoyl synthase (lipA), cation efflux system locus (cusA, cusB, cusF), lipid A palmitoyltransferase (pagP), cold shock-like protein (cspE), putative fluoride ion transporter $(c r c B)$, Sec-independent protein translocase (tatE), 2-oxoglutaramate amidase (yafV), and two hypothetical proteins.

Chemical and genetic rescue reveals causal SCV lesions. To determine which of the above lesions were responsible for the defects in growth observed in vitro, we performed chemical and genetic rescue experiments on the three SCV clones. We also tested the ability of the NCV isolates to rescue SCV growth by crossfeeding to further confirm that a diffusible factor was responsible for limited growth. All experiments were performed on M9 minimal medium.

Escherichia coli SCV prototrophic growth was restored with overexpression of hemL, but not hemF, fepC, or mprA (Fig. 2a). SCV growth was also rescued by the presence of heme (Fig. 2b) or $\delta$-aminolevulinic acid (ALA) (Supplementary Figure S1), but not L-glutamate, lipoic acid, or pyrroloquinolone quinone (PQQ) (Supplementary Figure S1). The SCV clone was also able to cross-feed from NCV (Fig. 2c). These results are all consistent with the genetic deletion in hemL being responsible for the limited growth in our E. coli clone.

SCVs often revert to the NCV phenotype when serially passaged in vitro. We identified an E. coli SCV that reverted to a normal growth phenotype over the course of our study. We performed WGS on this SCV revertant to understand the individual mutations that resulted in wild type growth. This isolate had an NCV-like hemL sequence, but retained the SCV coding mutations in hemF, fepC and $m p r A$. No additional mutations were observed in the reverted isolate. This further supports the results of our chemical and genetic rescues, and further highlights the importance of an intact hemL for normal growth.

Genetic and chemical rescue of $C$. freundii SCV growth showed similar results. C. freundii SCV prototrophic growth was restored with overexpression of hemL or fes, but not araC or pta (Fig. 3a). The same chemical rescue results were seen as in the E. coli SCV, as heme (Fig. 3b) or ALA (Supplementary Figure S2) were able to restore growth, but L-glutamate, lipoic acid, or PQQ failed to do so (Supplementary Figure S2). Moderate cross-feeding rescue with co-culture with the NCV clone was observed (Fig. $3 \mathrm{c}$ ). These results also indicate that deficiencies in heme synthesis and iron transport were responsible for the small colony growth phenotype seen in our $C$. freundii isolate.

Over the course of the rescue experiments, the $C$. freundii SCV also reverted to normal growth. This clone had NCV-like fes and hemL sequence, while the same SCV coding mutations were seen in $\operatorname{araC}, f d n G$, and pta, as well as the intergenic mutation upstream of hemF. The $C$. freundii revertant also had a new $\mathrm{R} 100 \mathrm{H}$ mutation in the cytochrome bd-I ubiquinol oxidase subunit 2 gene (CydB) relative to both the SCV and NCV clones. 


\begin{tabular}{|c|c|c|c|c|c|c|c|}
\hline Location & CDS codon number & CDS position & Nucleotide change & Protein effect & Coverage & Product & $\begin{array}{l}\text { Genetic restoration on } \\
\text { M9? }\end{array}$ \\
\hline \multicolumn{8}{|l|}{ Escherichia coli } \\
\hline hemF & ER232- & 694 & -GAGCGC & Deletion & 142 & $\begin{array}{l}\text { Coproporphyrinogen-III } \\
\text { oxidase, aerobic }\end{array}$ & No \\
\hline hemL & W59R & 175 & $\mathrm{~A}->\mathrm{T}$ & Substitution & 117 & $\begin{array}{l}\text { Glutamate-1-semialdehyde } \\
\text { 2,1-aminomutase }\end{array}$ & Yes \\
\hline fepC & E169X & 505 & $\mathrm{C}->\mathrm{A}$ & Truncation & 132 & $\begin{array}{l}\text { putative siderophore trans- } \\
\text { port system ATP-binding } \\
\text { protein YusV }\end{array}$ & No \\
\hline mprA & P84S & 250 & G->A & Substitution & 147 & $\begin{array}{l}\text { Transcriptional repressor } \\
\text { MprA }\end{array}$ & No \\
\hline \multicolumn{8}{|l|}{ Citrobacter freundii } \\
\hline $181 \mathrm{bp}$ upstream of hemF & $\mathrm{N} / \mathrm{A}$ & $\mathrm{N} / \mathrm{A}$ & $\mathrm{A}->\mathrm{C}$ & N/A & 26 & N/A & \\
\hline hemL & 18 & 54 & $-G$ & Frame Shift & 44 & $\begin{array}{l}\text { Glutamate-1-semialdehyde } \\
\text { 2,1-aminomutase }\end{array}$ & Yes \\
\hline fes & C306X & 918 & $\mathrm{~T}->\mathrm{A}$ & Truncation & 67 & Enterochelin esterase & Yes \\
\hline $\operatorname{araC}$ & P7L & 20 & $\mathrm{C}->\mathrm{T}$ & Substitution & 10 & $\begin{array}{l}\text { Arabinose operon regula- } \\
\text { tory protein }\end{array}$ & No \\
\hline $\mathrm{fdnG}$ & P778T & 2332 & $\mathrm{C}->\mathrm{A}$ & Substitution & 51 & \begin{tabular}{|l|} 
Formate dehydrogenase, \\
nitrate-inducible, major \\
subunit precursor
\end{tabular} & N.D \\
\hline pta & Q303L & 908 & $\mathrm{~T}->\mathrm{A}$ & Substitution & 72 & Phosphate acetyltransferase & No \\
\hline \multicolumn{8}{|l|}{ Enterobacter hormaechei } \\
\hline hemN & L366Q & 1097 & $A->T$ & Substitution & 34 & \begin{tabular}{|l} 
Oxygen-independent \\
coproporphyrinogen-III \\
oxidase 1
\end{tabular} & No \\
\hline pqqB & $\mathrm{C} 224 \mathrm{~W}$ & 672 & $\mathrm{C}->\mathrm{A}$ & Substitution & 57 & $\begin{array}{l}\text { Coenzyme PQQ synthesis } \\
\text { protein B }\end{array}$ & No \\
\hline entD & $\mathrm{D} 21 \mathrm{~A}$ & 62 & $\mathrm{C}->\mathrm{A}$ & Substitution & 44 & \begin{tabular}{|l|} 
4'-phosphopantetheinyl \\
transferase (enterobacin \\
biosynthesis complex)
\end{tabular} & No \\
\hline cysD & G291S & 871 & $\mathrm{~T}->\mathrm{C}$ & Substitution & 41 & $\begin{array}{l}\text { Sulfate adenylyltransferase } \\
\text { subunit } 2\end{array}$ & No \\
\hline lsrK & T403S & 1207 & A-> T & Substitution & 35 & Autoinducer 2 kinase LsrK & No \\
\hline $\operatorname{rcsC}$ & $\mathrm{I} 362 \mathrm{~T}$ & 1085 & G->A & Substitution & 38 & $\begin{array}{l}\text { Sensor histidine kinase } \\
\text { RcsC }\end{array}$ & No \\
\hline $\mathrm{udk}$ & $\mathrm{D} 74 \mathrm{H}$ & 220 & G->C & Substitution & 62 & Uridine kinase & No \\
\hline \multicolumn{8}{|c|}{ Deletions of the following genes } \\
\hline $\operatorname{lipA}$ & & & & & & Lipoyl Synthase & Yes \\
\hline cusF & & & & & & $\begin{array}{l}\text { Cation efflux system } \\
\text { protein }\end{array}$ & No \\
\hline cusB & & & & & & $\begin{array}{l}\text { Cation efflux system } \\
\text { protein }\end{array}$ & No \\
\hline cusA & & & & & & $\begin{array}{l}\text { Cation efflux system } \\
\text { protein }\end{array}$ & No \\
\hline pagP & & & & & & $\begin{array}{l}\text { Lipid A palmitoyltrans- } \\
\text { ferase }\end{array}$ & N.D \\
\hline $\operatorname{cspE}$ & & & & & & Cold shock-like protein & No \\
\hline $\operatorname{crcB}$ & & & & & & $\begin{array}{l}\text { Putative fluoride ion } \\
\text { transporter }\end{array}$ & No \\
\hline yafV & & & & & & 2-oxoglutaramate amidase & N.D \\
\hline tatE & & & & & & $\begin{array}{l}\text { Sec-independent protein } \\
\text { translocase protein }\end{array}$ & No \\
\hline Hypothetical protein & & & & & & Hypothetical protein & N.D \\
\hline Hypothetical protein & & & & & & Hypothetical protein & N.D \\
\hline
\end{tabular}

Table 2. Genomic variants isolated from paired clinical co-isolates of Escherichia coli, Citrobacter freundii, Enterobacter hormaechei.

These results further confirm the genetic and chemical rescue performed above, illustrating the importance of the heme pathway for normal growth.

Finally, despite also containing lesions in the heme biosynthesis pathway, the E. hormaechei SCV produced a radically different pattern of chemical and genetic rescue. Here, overexpression of lipA was the only gene that yielded prototrophic growth (Fig. 4a), while all other disrupted genes failed to rescue growth (Fig. 4a, Supplementary Figure S3). Complementation with lipoic acid restored growth (Fig. 4b) along with co-culture with $E$. 


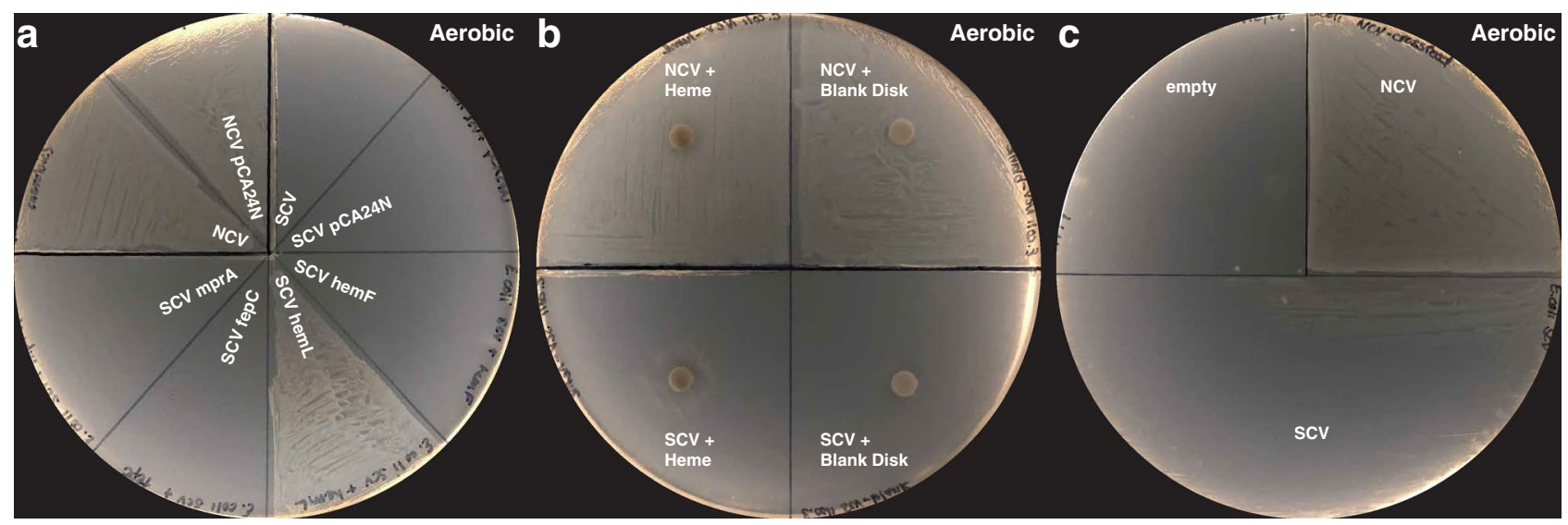

Figure 2. Heme biosynthesis lesion as cause of small colony phenotype in Escherichia coli isolated from urinary tract infection. Genomic sequencing of the paired NCV and SCV isolates revealed genomic lesions in fepC, hemF, hemL, and $m p r A$. (a) Only genetic rescue with hemL rescued normal growth from the Escherichia coli SCV. (b) Chemical rescue with heme partially restored normal growth in Escherichia coli SCV. (c) Cross-feeding from Escherichia coli NCV partially restores growth of SCV, consistent with a diffusible factor required for growth. All plates in this figure were incubated under aerobic conditions.

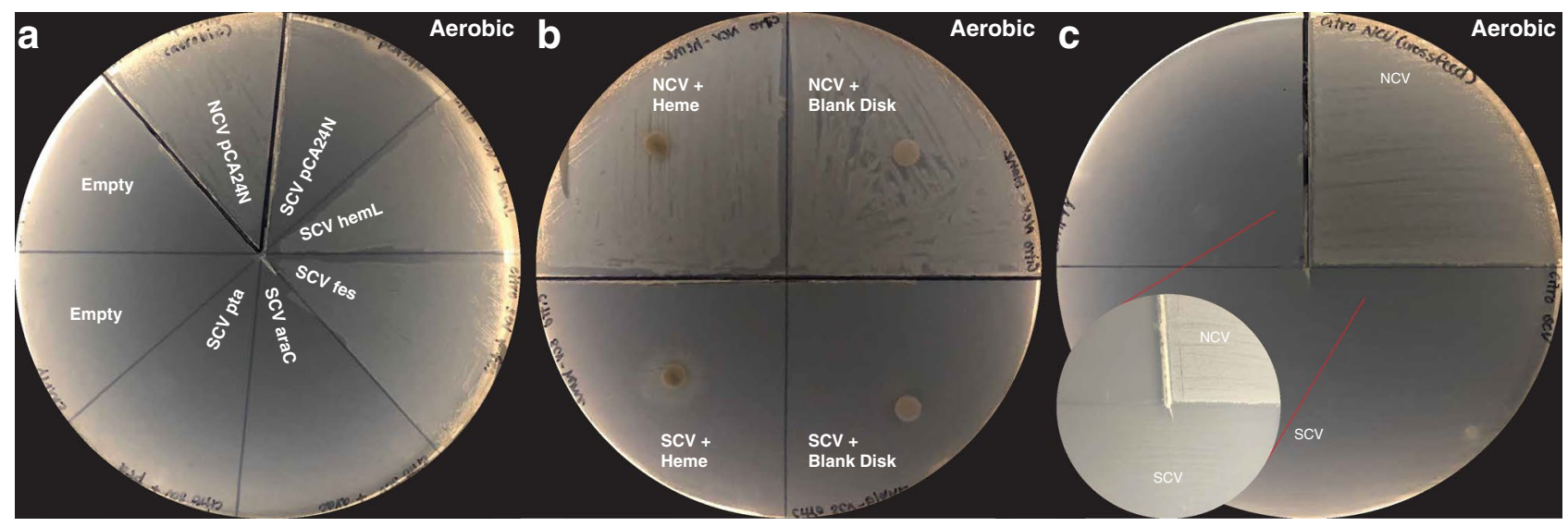

Figure 3. Heme biosynthesis along with iron availability lesion as cause of small colony phenotype in Citrobacter freundii isolated from urinary tract infection. Genomic sequencing of the paired NCV and SCV isolates revealed genomic lesions in $a r a C, f d n G$, fes, hemL, pta, and in the intergenic region upstream of the hemF gene. (a) Genetic rescue with fes and hemL rescued normal growth from the Citrobacter freundii SCV. (b) Chemical rescue with heme restored normal growth in Citrobacter freundii SCV. (c) Cross-feeding from Citrobacter freundii NCV partially restores growth of SCV, consistent with a diffusible factor required for growth. All plates in this figure were incubated under aerobic conditions.

hormaechei NCV (Fig. 4c), while PQQ, heme and its biosynthetic intermediates L-glutamate and ALA failed to increase SCV growth (Supplementary Figure S3). These results conclusively demonstrate that disruption of the lipoylation pathway was responsible for the small growth phenotype in our E. hormaechei SCV isolate. We did not observe reversion to normal growth for the E. hormaechei SCV.

Genetic rescue of the heme or lipoic acid biosynthesis pathway restores the growth kinetics of SCVs. In addition to unique colony morphologies and auxotrophy, a hallmark of the SCV phenotype is diminished growth kinetics compared to their NCV counterparts. We performed a growth curve analysis using the NCV and SCV co-isolates and the genetically complemented SCV strains which displayed prototrophic growth on M9 media: hemL for E. coli, hemL and fepC for C. freundii, and lipA for E. hormaechei. The E. coli and C. freundii SCV isolates both displayed an extended lag phase and exhibited a lower $\mathrm{OD}_{600}$ in the stationary phase compared to their NCV co-isolates (Fig. 5a,b). The E. hormaechei SCV did not displayed an extended lag phase compared its NCV co-isolate (Fig. 5c). However, the E. hormaechei SCV exited the exponential growth phase earlier than its NCV co-isolate and exhibited a lower $\mathrm{OD}_{600}$ during the stationary phase.

The growth kinetics of the E. coli SCV and C. freundii SCV isolates were completely rescued through overexpression hemL (Fig. 5a,b). Genetic complementation with fes did not rescue the growth kinetic of the C. freundii isolate. The growth kinetics of the E. hormaechei SCV isolate were partially restored by complementation with 


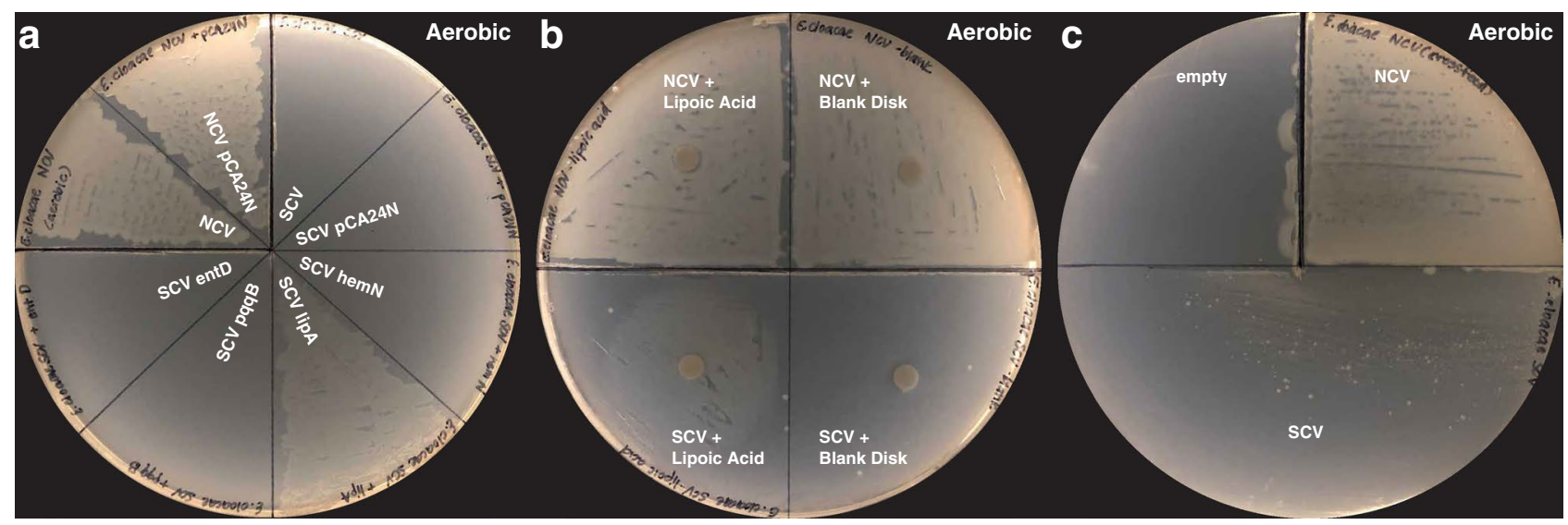

Figure 4. Lipoic acid biosynthesis as cause of small colony phenotype in Enterobacter hormaechei isolated from a bloodstream infection in a patient with end-stage renal disease. Genomic sequencing of the paired NCV and SCV isolates revealed multiple genomic lesions including single nucleotide substitutions in entD, hem $N$, and $p q q B$ along with large-scale rearrangements leading to disruption of the lipA gene. (a) Genetic rescue with lipA restored normal growth from the Enterobacter hormaechei SCV. (b) Chemical rescue with lipoic acid restored normal growth in Enterobacter hormaechei SCV. (c) Cross-feeding from Enterobacter hormaechei NCV restores growth of SCV, consistent with a diffusible factor required for growth. All plates in this figure were incubated under aerobic conditions.

a

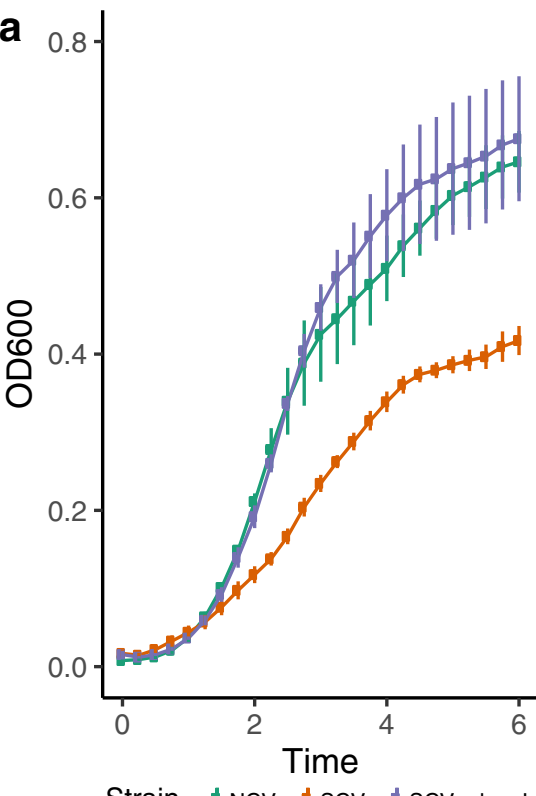

b

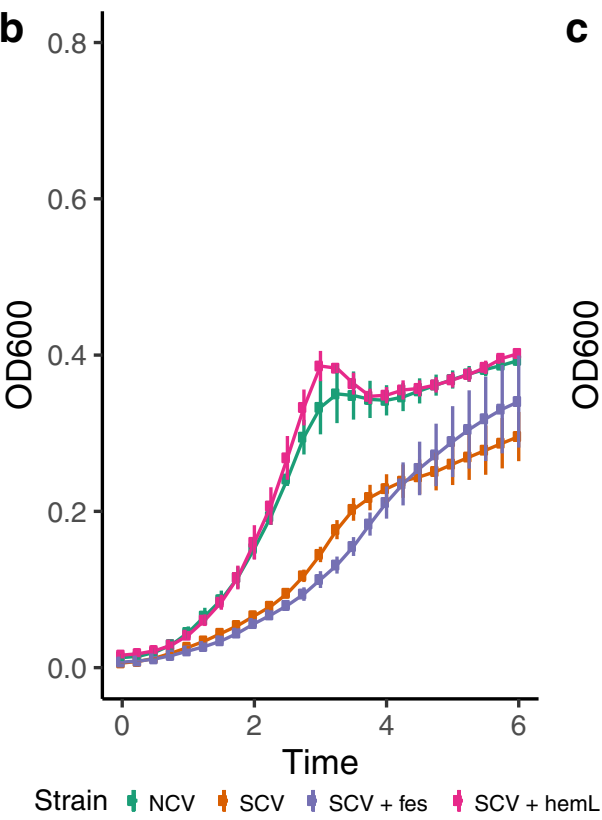

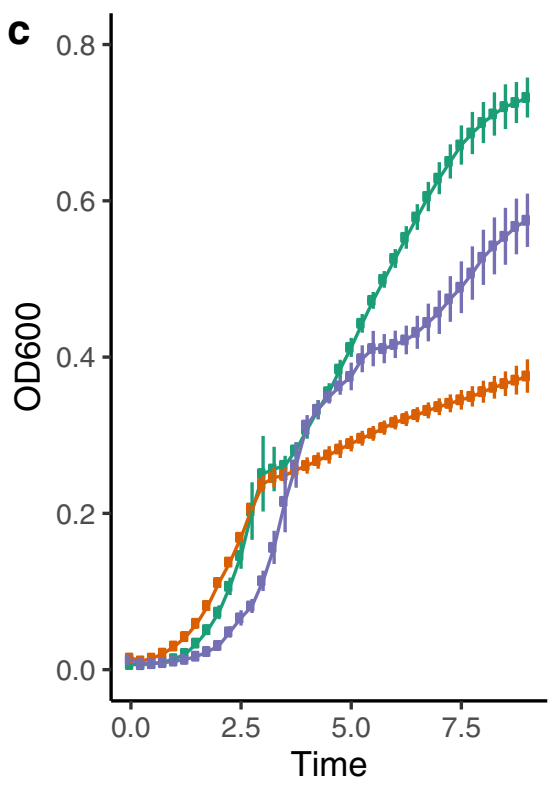

Strain $\& \mathrm{NCV} \& \mathrm{SCV} \& \mathrm{SCV}+$ lipA

Figure 5. Growth curve analysis in aerobic conditions demonstrates hemL fully restores the impaired growth kinetics of (a) Escherichia coli SCV and (b) Citrobacter freundii SCV isolates. The Citrobacter freundii SCV isolate was not rescued by fes. (c) Growth kinetics of the Enterobacter hormaechei SCV isolate are partially restored by overexpression of lipA.

lipA (Fig. 5c); however, the genetically complemented strain still exited the exponential growth phase earlier than the NCV isolate and exhibited a lower $\mathrm{OD}_{600}$ during the stationary phase.

SCVs demonstrate prototrophic growth and unaltered growth kinetics under anaerobic condi-

tions. Based on recurrent isolation of mutants in aerobic respiration pathways, along with the genetic and chemical rescue experiments demonstrating their causality, we hypothesized that SCV isolates might not demonstrate growth defects under anaerobic conditions. We cultured NCV and SCV isolates for each of the three species along with the transformed genetic rescue clones under anaerobic conditions for $48 \mathrm{~h}$. Anaerobic conditions rescued SCV growth in all cases (Figs. $6 \mathrm{a}-\mathrm{c}, \mathrm{S3h}-\mathrm{j}$ ). These results were also independent of every genetic construct transformed.

We next performed a growth curve analysis under anaerobic conditions with the NCV and SCV co-isolates. For all three pairs, similar growth kinetics were displayed between the NCV and SCV co-isolates (Fig. 6d-f). 


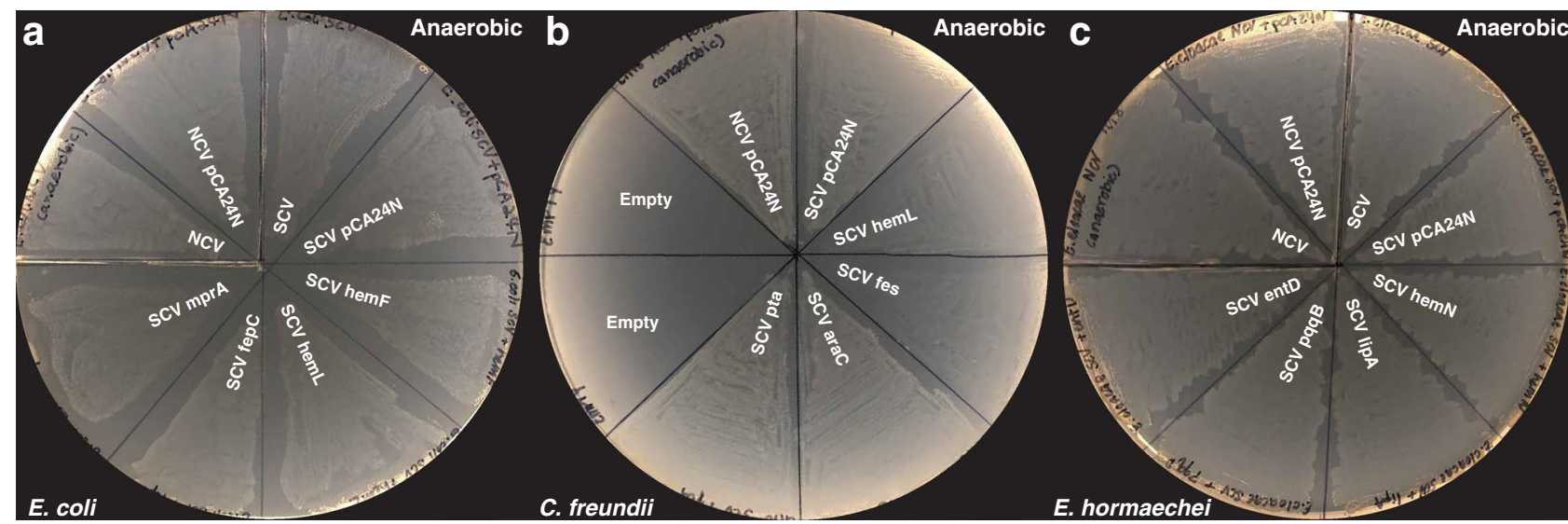

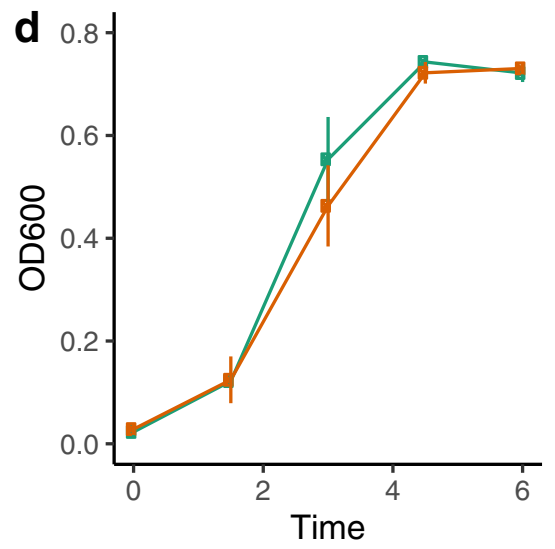

Strain $* \mathrm{NCV} * \mathrm{SCV}$

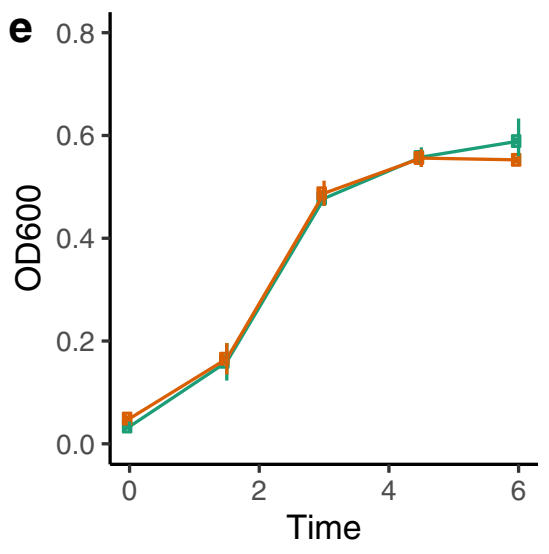

Strain * NCV \& SCV

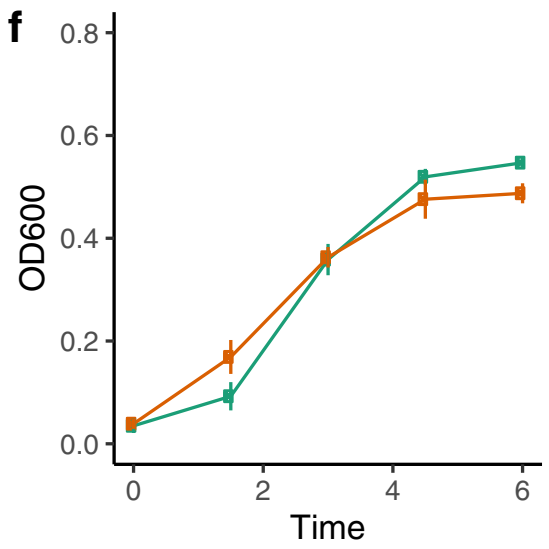

Strain NCV * SCV

Figure 6. SCV E. coli (a), C. freundii (b), and E. hormaechei (c) isolates grow on minimal media without additional chemical or genetic supplementation in anaerobic conditions. NCV and SCV E. coli (d), C. freundii (e), and E hormaechei (f) co-isolates display similar growth kinetics in anaerobic conditions.

\section{Discussion}

Here, we used genomic screening of paired isolates to understand the molecular mechanism of the small colony growth phenotype in Gram-negative bacteria encountered in the clinical microbiology laboratory as well as bacterial persistence in vivo. Unlike the NCVs, all three corresponding bacterial SCV isolates were auxotrophic, thus unable to grow in glucose only M9 medium. Using genetic, chemical rescue, as well as NCV cross-feeding, we found heme-production pathway lesions to be responsible for the SCV phenotype in two of the three isolates, while lipoic acid synthesis was responsible for the third. In the E. coli SCV, both W59R in hemL and two-amino acid deletion in hemF could impact heme production, but only complementation of hemL rescued growth on M9 media. In the SCV C. freundii, the truncation of hemL blocked ALA production. In E. hormaechei SCV, the lipA gene was interrupted by large-scale genomic deletion and, correspondingly, complementation with lipA or lipoic acid restored prototrophic growth. Each of the SCV isolates displayed diminished growth kinetics compared to the corresponding NCV co-isolate. We demonstrated the growth kinetics of the E.coli SCV and C. freundii SCV isolates could both be completed restored by overexpression of hemL, while the growth kinetics of E. hormaechi were only partially restored by overexpression of lipA. Intriguingly, growth of the SCVs was not impaired under anaerobic conditions, consistent with the role of heme as an essential cofactor for the electron transport chain and lipoic acid's role in the Krebs cycle. Of note, this is the first report of whole genome comparison of paired NCV and SCV Enterobacteriaceae isolates associated with clinical bloodstream and urinary tract infections from pediatric patients and the first report of detection of gene lesions associated with bacterial iron acquisition. Gene truncations in enterochelin esterase ( $f e s)$ in the SCV C. freundii, a putative siderophore transport system ATPbinding protein $(f e p C)$ in the SCV E. coli, and D21A in entD of E. hormaechei were novel findings with potential implications for bacterial lifestyle changes upon host selection. Notably, despite the significant genetic lesions recovered here-two truncations and a coding mutation never previously recovered in any Enterobacter hormaechei-complementation with $f e p C$ and $e n t D$ genes from $E$. coli failed to rescue prototrophic growth. Future work will need to characterize the effect of these mutations on protein function, as it is possible that these mutations result in dominant negative phenotypes.

The antibiotic exposure history or the choices for certain specific agent(s) used for the treatment in the 3 patients at the time could not explain a common pattern of selective pressure for the emergence of bacterial SCV. The patient underlying illnesses also ranged from the first episode of a potential E. coli urosepsis in a newborn, 
a recurrent $C$. freundii UTI in a 2-month old, and a presumed $E$. hornaechei occult renal system infection in a 6-year old patient with end stage renal disease pending kidney transplant. The second and the third cases both had significant prior antimicrobial exposure to multiple classes of agents with beta-lactams being the most common agent, but first new born case had no prior treatment ever. Although prior exposure to aminoglycosides or sulfamethoxazole-trimethoprim has been reported as selective pressure for selection of bacterial SCVs ${ }^{4,29}$, these two agents were only used in the third case. Therefore, antibiotic use alone may not be the major contributor for SCV selection.

Pairwise sequencing of the NCV and SCV genomes for SCV mutational characterization was based on the assumption that both NCV and SCV descended from the same strain. While the NCV is likely the closest representative of the parental strain, SCV diverged from this strain with distinctive mutations that were absent in NCV. The functional M9 growth studies in vitro have clearly demonstrated that deficiencies in bacterial synthesis of heme, lipoic acid, and/or iron acquisition apparatus were the primary contributors to SCV auxotrophism. Our analysis suggests that both the oxidative respiration and iron acquisition may be counter-selected by the host during the subacute or perhaps chronic infections. Moreover, the extent of SCV genomic mutations could vary depending on the clinical course of the infective illnesses. For example, the E. coli SCV urinary tract isolate from a 6 week old infant had 4 SNVs, the repeat $C$. freundii SCV urinary tract isolate from a 2 month old patient with urological anomalies had 6 SNVs, and the blood stream E. hormaechei SCV isolate from a 6 year old patient with end stage renal disease had 8 SNVs plus an 11-gene deletion.

The selective loss of oxidative respiration and iron acquisition we observed in the SCV isolates sharply contrasts with conventionally held beliefs about microbial pathogens. Rather than rapidly dividing and completing fiercely for iron, SCVs take a unique approach for evading the host defense response, which includes iron starvation and oxidative stress ${ }^{1,2}$. In response to the iron-limiting condition, it has been well documented that the bacterial ferric uptake regulator (Fur) system is activated to strengthen microbial ability to capture iron for energetic growth and pathogenesis ${ }^{3}$. Regardless of iron availability, Enterobacteriaceae are facultative anaerobes, and they are fully capable of growth under various oxygen tensions with oxidative respiration being significantly more robust for energetic growth in vitro ${ }^{30}$. Therefore, change in oxygen tension itself in the infected host environment may not be the major selective pressure for SCV development. Our finding of mutations in oxidative respiration related genes was consistent with the overwhelming reports of bacterial SCV deficiencies in heme, menaquinone, and lipoic acid synthesis ${ }^{4,21,31,32}$. Thus, an overarching impression of the SCV degenerative mutations is their association with the essential elements of aerobic living which demands more iron ${ }^{33}$. The SCV isolates in this study, regardless of heme, lipoic acid, and/or iron transport deficiencies, were all able to grow anaerobically on M9 without nutrient supplementation. In fact, the selective loss of aerobic respiration under iron limiting and oxidative stress conditions is indicative of a microbial survival mechanism by "retreating to anaerobic habitats" in order to persist inside a single affected host without clonal dissemination into the host population ${ }^{4,34-36}$. Hence, resorting to anaerobic living may be an alternative response to iron limitation and oxidative stress ${ }^{37}$. This alternative survival mechanism would be opposite to the well-known "superbugs" survival strategy of head-on iron competition and host population dissemination ${ }^{38,39}$. It also shares similarities with the immunoevasion strategies of tumors via hypoxia-induced immune exhaustion and T-cell anergy ${ }^{40}$.

Iron is an essential nutrient for all forms of life ${ }^{41}$. However, some free-living and obligate parasitic bacteria (e.g. Borrelia burgdorferi) employ a unique iron-independent redox-active metal such as manganese (Mn) to deal with oxidative stress, which allows them to bypass host iron defense ${ }^{42}$. E. coli maintains a Mn-SOD (superoxide dismutase) system which is also regulated by Fur ${ }^{43}$. All three SCV isolates contained an intact sodA, though expression and functional anti-oxidant activities were not examined. It is conceivable that the inactivation of iron uptake apparatus in bacterial SCVs may be indicative of bacterial transition into a parasitic lifestyle by means of iron bypass ${ }^{42}$. Alternatively, the genetic lesions described here, particularly in E. hormaechei, could also be indicative of evolution to a more symbiotic lifestyle, as evidenced by the cross-feeding and gene decay ${ }^{44}$. The co-obligate symbiotic bacterium Serratia symbiotica, known for its minimal genome $(\sim 143 \mathrm{~kb})$, has lost the ability to synthesize both heme and enterobactin and, additionally, lacks an iron uptake apparatus, similar to the changes observed in our SCV isolates ${ }^{45,46}$.

This study is limited by our focus only on mutations that were associated with auxotrophism on M9 medium without analyzing other mutations that could potentially affect many other aspects of bacterial functions. For example, a 10.2-kb deleted fragment in E. hormaechei SCV contained cusF, cusB, and cusA in tandem where CusCFBA is a membrane-bound proton antiporter for $\mathrm{Cu} / \mathrm{Ag}$ efflux ${ }^{47}$. As this efflux system carries out ATPdemanding activities, we speculate that this function encounters the same counter-selective pressure as a result of reduced ATP production assuming SCV anaerobic lifestyle. The exact host factor(s) that was critical for selection of SCV mutations is still unknown. Ideally, host selective factors could be identified if we could recreate the bacterial SCVs from the wildtype using host materials for bacterial growth in vitro or in animal models.

Antimicrobial resistance and iron competition as mechanisms for bacterial survival have attracted a great deal of interest ${ }^{48}$. These isolates are now readily recognized in the clinical microbiology laboratory due to their robust growth and detectable resistance phenotypes using standard in vitro testing. Clinical isolates such as SCVs that lack these growth characteristics may be easily missed due to their small colony size and failure to grow on standard susceptibility testing media. The role of these alternatively pathways of survival in clinical infections may be significantly underestimated ${ }^{32}$. Future work must address the totality of bacterial survival mechanisms, including mechanisms of bacteria persistence via resistance to host iron immunity and oxidative stress by means of anaerobiosis and iron bypass.

\section{Data availability}

All genome assemblies are available from NCBI BioProject PRJNA523376. 
Received: 24 September 2020; Accepted: 11 March 2021

Published online: 02 April 2021

\section{References}

1. Cassat, J. E. \& Skaar, E. P. Iron in infection and immunity. Cell Host Microbe 13, 509-519 (2013).

2. Skaar, E. P. The battle for iron between bacterial pathogens and their vertebrate hosts. PLoS Pathog. 6, e1000949 (2010).

3. Porcheron, G. \& Dozois, C. M. Interplay between iron homeostasis and virulence: Fur and RyhB as major regulators of bacterial pathogenicity. Vet. Microbiol. 179, 2-14 (2015).

4. Proctor, R. A. et al. Small colony variants: A pathogenic form of bacteria that facilitates persistent and recurrent infections. Nat. Rev. Microbiol. 4, 295-305 (2006).

5. Grant, S. S. \& Hung, D. T. Persistent bacterial infections, antibiotic tolerance, and the oxidative stress response. Virulence 4, 273-283 (2013).

6. Garcia, L. G. et al. Antibiotic activity against small-colony variants of Staphylococcus aureus: Review of in vitro, animal and clinical data. J. Antimicrob. Chemother. 68, 1455-1464 (2013).

7. Kahl, B. C., Becker, K. \& Löffler, B. Clinical significance and pathogenesis of staphylococcal small colony variants in persistent infections. Clin. Microbiol. Rev. 29, 401-427 (2016).

8. Anderson, S. W., Stapp, J. R., Burns, J. L. \& Qin, X. Characterization of small-colony-variant stenotrophomonas maltophilia isolated from the sputum specimens of five patients with cystic fibrosis. J. Clin. Microbiol. 45, 529-535 (2007).

9. Wellinghausen, N. et al. Characterization of clinical Enterococcus faecalis small-colony variants. J. Clin. Microbiol. 47, 2802-2811 (2009).

10. Roggenkamp, A. et al. Chronic prosthetic hip infection caused by a small-colony variant of Escherichia coli. J. Clin. Microbiol. 36, 2530-2534 (1998).

11. Săsărman, A., Sanderson, K. E., Surdeanu, M. \& Sonea, S. Hemin-deficient mutants of Salmonella typhimurium. J. Bacteriol. 102, 531-536 (1970).

12. Morton, H. E. \& Shoemaker, J. The Identification of Neisseria gonorrhoeae by means of bacterial variation and the detection of small colony forms in clinical material. J Bacteriol 50, 585-587 (1945).

13. Kriegeskorte, A. et al. Inactivation of thyA in Staphylococcus aureus attenuates virulence and has a strong impact on metabolism and virulence gene expression. MBio 5, e01447-e11414 (2014).

14. Choby, J. E. \& Skaar, E. P. Heme synthesis and acquisition in bacterial pathogens. J. Mol. Biol. 428, 3408-3428 (2016).

15. Dean, M. A., Olsen, R. J., Long, S. W., Rosato, A. E. \& Musser, J. M. Identification of point mutations in clinical Staphylococcus aureus strains that produce small-colony variants auxotrophic for menadione. Infect. Immun. 82, 1600-1605 (2014).

16. Lannergård, J. et al. Identification of the genetic basis for clinical menadione-auxotrophic small-colony variant isolates of Staphylococcus aureus. Antimicrob. Agents Chemother. 52, 4017-4022 (2008).

17. Clements, M. O., Watson, S. P., Poole, R. K. \& Foster, S. J. CtaA of Staphylococcus aureus is required for starvation survival, recovery, and cytochrome biosynthesis. J. Bacteriol. 181, 501-507 (1999).

18. Pränting, M. \& Andersson, D. I. Escape from growth restriction in small colony variants of Salmonella typhimurium by gene amplification and mutation. Mol. Microbiol. 79, 305-315 (2011).

19. von Eiff, C. et al. A site-directed Staphylococcus aureus hemB mutant is a small-colony variant which persists intracellularly. J. Bacteriol. 179, 4706-4712 (1997).

20. Ramiro, R. S., Costa, H. \& Gordo, I. Macrophage adaptation leads to parallel evolution of genetically diverse Escherichia coli smallcolony variants with increased fitness in vivo and antibiotic collateral sensitivity. Evol. Appl. 9, 994-1004 (2016).

21. Santos, V. \& Hirshfield, I. The physiological and molecular characterization of a small colony variant of Escherichia coli and its phenotypic rescue. PLoS ONE 11, e0157578 (2016).

22. Sifri, C. D., Baresch-Bernal, A., Calderwood, S. B. \& von Eiff, C. Virulence of Staphylococcus aureus small colony variants in the Caenorhabditis elegans infection model. Infect. Immun. 74, 1091-1096 (2006).

23. Painter, K. L. et al. Staphylococcus aureus adapts to oxidative stress by producing H2O2-resistant small-colony variants via the SOS response. Infect. Immun. 83, 1830-1844 (2015).

24. Tada, T. et al. A hemin auxotrophic Enterobacter cloacae clinical isolate with increased resistance to carbapenems and aminoglycosides. J. Med. Microbiol. 67, 29-32 (2018).

25. Greninger, A. L. et al. Two rapidly growing mycobacterial species isolated from a brain abscess: First whole-genome sequences of mycobacterium immunogenum and mycobacterium llatzerense. J. Clin. Microbiol. 53, 2374-2377 (2015).

26. Addetia, A. et al. A novel, widespread qacA allele results in reduced chlorhexidine susceptibility in Staphylococcus epidermidis. Antimicrob. Agents Chemother. 63, e02607-e02618 (2019)

27. Greninger, A. L., Streithorst, J., Golden, J. A., Chiu, C. Y. \& Miller, S. Complete genome sequence of sequential Pandoraea apista isolates from the same cystic fibrosis patient supports a model of chronic colonization with in vivo strain evolution over time. Diagn. Microbiol. Infect. Dis. 87, 1-6 (2017).

28. Kitagawa, M. et al. Complete set of ORF clones of Escherichia coli ASKA library (a complete set of E. coli K-12 ORF archive): Unique resources for biological research. DNA Res. 12, 291-299 (2005).

29. Melter, O. \& Radojevič, B. Small colony variants of Staphylococcus aureus-Review. Folia Microbiol. (Praha) 55, 548-558 (2010).

30. Gonzalez, J. E., Long, C. P. \& Antoniewicz, M. R. Comprehensive analysis of glucose and xylose metabolism in Escherichia coli under aerobic and anaerobic conditions by 13C metabolic flux analysis. Metab. Eng. 39, 9-18 (2017).

31. von Eiff, C., Peters, G. \& Becker, K. The small colony variant (SCV) concept-The role of staphylococcal SCVs in persistent infections. Injury 37(Suppl 2), S26-33 (2006).

32. Park, Y. J. et al. Urinary tract infection caused by a small colony variant form of capnophilic Escherichia coli leading to misidentification and non-reactions in antimicrobial susceptibility tests. Antimicrob. Resist. Infect. Control 7, 139 (2018).

33. Beauchene, N. A. et al. Impact of anaerobiosis on expression of the iron-responsive fur and RyhB regulons. MBio 6, e01947-e11915 (2015).

34. Imlay, J. A. Iron-sulphur clusters and the problem with oxygen. Mol. Microbiol. 59, 1073-1082 (2006).

35. Qin, X. Chronic pulmonary pseudomonal infection in patients with cystic fibrosis: A model for early phase symbiotic evolution. Crit. Rev. Microbiol. 42, 144-157 (2016).

36. Proctor, R. A. et al. Staphylococcus aureus small colony variants (SCVs): A road map for the metabolic pathways involved in persistent infections. Front. Cell Infect. Microbiol. 4, 99 (2014).

37. Dukan, S. \& Nyström, T. Oxidative stress defense and deterioration of growth-arrested Escherichia coli cells. J. Biol. Chem. 274, 26027-26032 (1999)

38. Miller, H. K. \& Auerbuch, V. Bacterial iron-sulfur cluster sensors in mammalian pathogens. Metallomics 7, 943-956 (2015).

39. Peltier, F. et al. Characterization of a multidrug-resistant Klebsiella pneumoniae ST607-K25 clone responsible for a nosocomial outbreak in a neonatal intensive care unit. J. Med. Microbiol. 68, 67-76 (2019).

40. Noman, M. Z. et al. Hypoxia: A key player in antitumor immune response. A review in the theme: Cellular responses to hypoxia. Am. J. Physiol. Cell Physiol. 309, C569-579 (2015).

41. Cairo, G., Bernuzzi, F. \& Recalcati, S. A precious metal: Iron, an essential nutrient for all cells. Genes Nutr. 1, 25-39 (2006). 
42. Posey, J. E. \& Gherardini, F. C. Lack of a role for iron in the Lyme disease pathogen. Science 288, 1651-1653 (2000).

43. Fee, J. A. Regulation of sod genes in Escherichia coli: Relevance to superoxide dismutase function. Mol. Microbiol. 5, 2599-2610 (1991).

44. Gil, R., Sabater-Muñoz, B., Latorre, A., Silva, F. J. \& Moya, A. Extreme genome reduction in Buchnera spp.: Toward the minimal genome needed for symbiotic life. Proc. Natl. Acad. Sci. USA 99, 4454-4458 (2002).

45. Burke, G. R. \& Moran, N. A. Massive genomic decay in Serratia symbiotica, a recently evolved symbiont of aphids. Genome Biol. Evol. 3, 195-208 (2011).

46. Manzano-Marín, A. \& Latorre, A. Settling down: the genome of Serratia symbiotica from the aphid Cinara tujafilina zooms in on the process of accommodation to a cooperative intracellular life. Genome Biol. Evol. 6, 1683-1698 (2014).

47. Franke, S., Grass, G., Rensing, C. \& Nies, D. H. Molecular analysis of the copper-transporting efflux system CusCFBA of Escherichia coli. J. Bacteriol. 185, 3804-3812 (2003).

48. Broberg, C. A., Palacios, M. \& Miller, V. L. Klebsiella: A long way to go towards understanding this enigmatic jet-setter. F1000Prime Rep. 6, 64 (2014).

\section{Acknowledgements}

We thank National BioResource Project NBRP E.coli Strain, Japan for the E. coli plasmid library (https://shigen. nig.ac.jp/ecoli/strain/). We also thank Seattle Children's Microbiology team for their effort in standardization of bacterial SCV identification and quality documentation of these paired isolates.

\section{Author contributions}

Conceptualization-A.L.G., X.Q., Amin Addetia; data curation-A.L.G., Amin Addetia, Amanda Adler, X.Q.; formal analysis-A.L.G., Amin Addetia, X.Q.; funding acquisition-A.L.G., X.Q.; resources-A.L.G., X.Q.; investigation-A.L.G., Amin Addetia, Y.T.; methodology-A.L.G., Amin Addetia, X.Q.; project administrationA.L.G., X.Q., Amin Addetia; Visualization-A.L.G., Amin Addetia, X.Q.; supervision-A.L.G., X.Q.; validationA.L.G., Amin Addetia, X.Q.; writing-original draft-A.L.G., Amin Addetia, X.Q.; writing-reviewing and editing-all authors.

\section{Competing interests}

The authors declare no competing interests.

Additional information

Supplementary Information The online version contains supplementary material available at https://doi.org/ 10.1038/s41598-021-86764-4.

Correspondence and requests for materials should be addressed to X.Q.

Reprints and permissions information is available at www.nature.com/reprints.

Publisher's note Springer Nature remains neutral with regard to jurisdictional claims in published maps and institutional affiliations.

(c) (1) Open Access This article is licensed under a Creative Commons Attribution 4.0 International License, which permits use, sharing, adaptation, distribution and reproduction in any medium or format, as long as you give appropriate credit to the original author(s) and the source, provide a link to the Creative Commons licence, and indicate if changes were made. The images or other third party material in this article are included in the article's Creative Commons licence, unless indicated otherwise in a credit line to the material. If material is not included in the articles Creative Commons licence and your intended use is not permitted by statutory regulation or exceeds the permitted use, you will need to obtain permission directly from the copyright holder. To view a copy of this licence, visit http://creativecommons.org/licenses/by/4.0/.

(c) The Author(s) 2021 